\title{
ENTIRE FUNCTIONS WITH PRESCRIBED VALUES AT DISCRETE POINT SETS
}

\author{
BY \\ L. D. NEIDLEMAN( ${ }^{(1)}$
}

1. Introduction. Most of the work done, to date, on entire functions with prescribed values at discrete point sets has been restricted to those functions whose "value set" is the algebraic numbers or some subset of these. Quite a bit has been accomplished on integral valued entire functions. In particular, certain bounds for the order and type of these functions have been determined. See R. C. Buck [2], G. Pólya [4] and G. H. Hardy [3]. Order and type bounds have also been determined for entire functions which have all their derivatives specified as integral at integral points. See E. G. Straus [5] and D. Sato [6].

In this paper we will consider more general "value sets," $V$, and more general discrete point sets or "domain sets" at which all the derivatives of the entire functions under consideration take values in $V$. We will determine certain "critical points" for the order and type of these more generalized classes of entire functions. D. Sato and E. G. Straus [7] recently showed that it is possible to construct entire functions (in fact $2^{\aleph_{0}}$ of them) which have values and derivatives of all orders belonging to a given set $V$ of complex numbers, at all points of a given discrete set of points (that is, a set of points without finite limit points) as long as there is some $\varepsilon>0$ such that the distance of the complex point $z$ from $V$, is less than $|z|^{1-\varepsilon}$, for all sufficiently large $|z|$. Using similar conditions on our value sets, we will determine dividing lines for the order of these functions such that if the functions are of order less than a certain critical value there will only be a countable number of functions and another critical value such that there will be an uncountable number of functions in our family of any order greater than or equal to this critical value. Similar dividing lines for the types of these functions are also obtained. The dividing lines for the order and type are derived as functions of parameters which depend only on the "domain" and "value sets" under consideration.

2. Definitions and main results. We start with the definition of a generalized Taylor series.

DeFinition 1. Let $\left\{z_{k}\right\}$ be a sequence of complex numbers, then a series

$$
f(z)=\sum_{n=0}^{\infty} a_{n}\left(z-z_{1}\right)\left(z-z_{2}\right) \cdots\left(z-z_{n}\right)
$$

is called a generalized Taylor series (GTS).

Received by the editors October 24, 1967 and, in revised form, June 20, 1968 and September 23, 1968.

( ${ }^{1}$ This research was done while the author was writing his Ph.D. dissertation. The research was sponsored in part by a Howard Hughes Staff Doctoral Fellowship. 
For generalized Taylor series at a bounded sequence the usual expressions (cf. Boas [1]) for the order and type of an entire function remain valid.

THEOREM 1. Let $f(z)=\sum a_{n}\left(z-z_{1}\right) \cdots\left(z-z_{n}\right)$ be an entire function where $\left\{z_{n}\right\}$ is bounded. Then the order $\rho$ of $f(z)$ is given by

$$
\rho=\limsup _{n \rightarrow \infty} \frac{n \log n}{\log \left|a_{n}\right|^{-1}}
$$

and the type $\sigma$ of $f(z)$ is given by

$$
\sigma=\frac{1}{e \rho} \limsup _{n \rightarrow \infty} n\left|a_{n}\right|^{\rho / n} .
$$

In this paper, the generalized Taylor series representations for our entire functions will have the form

$$
f(z)=\sum_{n=0}^{\infty} a_{n} \prod_{i=1}^{\infty}\left(z-z_{i}\right)^{m_{i}}
$$

with $\sum m_{i}=n$ so that for all $i>H(n), m_{i}=0$.

We use the representation (7) so that we can relate the coefficients to the order and type of our entire functions as in Theorem 1, and then determine order and type bounds for classes of entire functions as mentioned earlier. We will be concerned with the values of $f(z)$ and its derivatives at the $z_{i}$ in (7).

For later reference we therefore make the following definitions:

Definition 2. A domain set, $D$, of the complex plane, $C$, is a (countable) set without finite limit points.

Definition 3. If $f^{(n)}(z) \in V$ for $n=0,1,2, \ldots$, and all $z \in D$ then $V$ is called a value set of $f(z)$ on $D$.

We also define the following "critical points" for both the order and type.

Definition 4. Let $\mathscr{F}$ be a family of entire functions, let $\mathscr{F}_{o}=\{f \mid f \in \mathscr{F}$, order $f \leqq \rho\}$, and let

$$
\mathscr{F}_{\rho}^{*}=\{f \mid f \in \mathscr{F}, \text { order } f<\rho\}=\bigcup_{\rho^{\prime}<\rho} \mathscr{F}_{\rho^{\prime}} .
$$

$\rho_{0}$ is a countable critical point for $\mathscr{F}$ if and only if $\mathscr{F}_{\rho_{0}}^{*}$ is countable. $\rho_{1}$ is an uncountable critical point for $\mathscr{F}$ if $\mathscr{F}_{\rho_{1}}$ is uncountable. If $\mathscr{F}$ is such that $\rho_{0}=\rho_{1}$ then $\rho^{*}=\rho_{0}=\rho_{1}$ is the critical point for $\mathscr{F}$. We let $\mathscr{F}$ be a family of entire functions of order $\rho$ and make similar definitions for the critical points $\sigma_{0}, \sigma_{1}$, and $\sigma^{*}$ for the types of the elements of $\mathscr{F}$.

The next theorem we give is a slight generalization of D. Sato's Theorem 2-6-I [6]. This result is given mainly because we will later use some of the same techniques and terminology.

THEOREM 2. Let $\mathscr{F}$ be the family of entire functions with a finite domain set, $D \subset R$ (the reals), consisting of $k$ points and suppose the value set for all $f \in \mathscr{F}$ on $D$ is the integers, I. Then $\rho^{*}=k$ and $\sigma^{*}=\left|V_{k}\right|^{-2 / k}$ where $V_{k}=\prod_{i>j}^{k}\left(z_{i}-z_{j}\right)$. 
THEOREM 3. Suppose $\mathscr{F}$ has a domain set, $D$, consisting of $k$ points and the value set for $\mathscr{F}$ is the Gaussian integers, $I_{G}$. Then $\rho^{*}=k$ and $\sigma^{*}=\left|V_{k}\right|^{-2 / k}$.

THEOREM 4. Let $\mathscr{F}$ be the family of entire functions with finite domain set, $D$, consisting of $k$ points and a countable value set, $V$, which satisfies

$$
\left|v-v^{\prime}\right|>c_{1} /(1+|v|)^{\lambda}
$$

$\lambda \geqq 0, c_{1}>0$ for all $v \neq v^{\prime}$. Then $\rho_{0}=(k+\lambda) /(1+\lambda)$ is a countable critical point of $\mathscr{F}$.

Proof. Let $f(z)$ have the generalized Taylor series

$$
f(z)=\sum_{s=0}^{\infty} a_{s} P_{s}(z)
$$

where $P_{s}(z)=\prod_{i=1}^{k}\left(z-z_{i}\right)^{s_{i}}, s_{1}+\cdots+s_{k}=s$, and $s_{l}=s_{l}(s)=[s / k]$.

Taking the $s_{l}$ th derivative of $f(z)$ at $z_{l}$ gives

$$
f^{\left(s_{l}\right)}\left(z_{l}\right)=a_{s} s_{l} ! \prod_{i=1 ; i \neq l}^{k}\left(z_{l}-z_{i}\right)^{s_{i}}+R_{s}
$$

where

$$
R_{s}=\frac{d^{s_{l}}}{d z^{s_{l}}}\left[\sum_{v=0}^{s-1} a_{v} P_{v}(z)\right]_{z=z_{l}}
$$

In other words, $a_{s}$ is the highest coefficient remaining when $z_{l}$ is substituted into $f^{\left(s_{l}\right)}(z)$. From (4.3) we get

$$
R_{s}=\sum_{\nu=s_{l}}^{s-1} a_{v} s_{l} ! \sum_{\sum \lambda_{i}=s_{l} ; \sum \nu_{l}=v} \prod_{i \neq l}^{k}\left(\begin{array}{l}
\nu_{i} \\
\lambda_{i}
\end{array}\right)\left(z_{l}-z_{i}\right)^{\nu_{i}-\lambda_{i}} .
$$

We now seek an upper bound for $\left|R_{s}\right|$.

$$
\left|R_{s}\right| \leqq \sum_{\nu=s_{l}}^{s-1}\left|a_{v}\right| s_{l} ! \sum_{\Sigma \lambda_{i}=s_{l}} \prod_{i \neq l}^{k}\left(\begin{array}{c}
\nu_{i} \\
\lambda_{l}
\end{array}\right)\left|z_{l}-z_{l}\right|^{v_{i}-\lambda_{i}}
$$

Let $f(z)$ have order $\rho$, then for every $\varepsilon>0$ there is a $c_{2}$ so that

$$
\left|a_{v}\right| \leqq \frac{c_{2}}{v^{v /(0+8)}}
$$

Now since

$$
\sum_{\sum \lambda_{i}=s_{l}} \prod_{i \neq l}^{k}\left(\begin{array}{c}
\nu_{i} \\
\lambda_{i}
\end{array}\right)\left|z_{l}-z_{i}\right|^{\nu_{i}-\lambda_{i}}<c_{3}^{s}
$$

(4.5), (4.6), and (4.7) imply

$$
\left|R_{s}\right| \leqq \sum_{\nu=s_{l}}^{s-1} \frac{c_{2} s_{l} ! c_{3}^{s}}{\nu^{v /(\rho+\varepsilon)}}
$$

By maximizing the summand of (4.8) we find the maximum occurs at $\gamma_{0}=1 / e$. 
For all $\nu \geqq \gamma_{0}$, the summand is a strictly decreasing function of $\nu$, hence for $s$ sufficiently large we get

$$
\left|R_{s}\right| \leqq c_{4} s s_{l} ! c_{3}^{s} / s_{l}^{s_{l} /(\rho+\varepsilon)}
$$

Using $s_{l}=[s / k]$ we get

$$
\left|R_{s}\right| \leqq s^{(s / k)(1-1 /(\rho+\varepsilon))} e^{O(s)}=\mathscr{R}_{s}
$$

Since $f^{(s)}\left(z_{l}\right)=v_{s}$ is to be in $V,(4.2)$ gives

$$
\left|v_{s}-R_{s}\right|=\left|a_{s} s_{l} ! \prod_{i \neq l}^{k}\left(z_{l}-z_{i}\right)^{s_{i}}\right|=s^{s / k}\left|a_{s}\right| e^{O(s)}<s^{s / k-s /(\rho+\varepsilon)} e^{O(s)} .
$$

Now, for $c_{5}$ sufficiently small, the inequality

$$
\left|v_{s}-R_{s}\right|<c_{5} / \mathscr{R}_{s}^{\lambda}, \quad v_{s} \in V
$$

determines $v_{s}$ uniquely, if it can be solved at all. $\left(\mathscr{R}_{s} \rightarrow \infty\right.$ as $s \rightarrow \infty$.) I.e., if there exist two solutions $v_{s}$ and $v_{s}^{\prime} \in V$ satisfying (4.12) then

$$
\left|v_{s}-v_{s}^{\prime}\right|<2 c_{5} \mid \mathscr{R}_{s}^{\lambda}
$$

Inequality (4.12) implies

$$
\left|v_{s}\right|<\left|R_{s}\right|+c_{5} \mid \mathscr{R}_{s}^{\lambda} \leqq \mathscr{R}_{s}+c_{5} / \mathscr{R}_{s}^{\lambda}<\left(c_{5}+1\right) \mathscr{R}_{s} .
$$

By hypothesis we have

$$
\left|v_{s}-v_{s}^{\prime}\right|>c_{1} /\left(1+\left|v_{s}\right|\right)^{\lambda}
$$

so that

$$
2 c_{5}\left|\mathscr{R}_{s}^{\lambda}>\right| v_{s}-v_{s}^{\prime} \mid>c_{1} /\left(1+\left(c_{5}+1\right) \mathscr{R}_{s}\right)^{\lambda}>c_{1} /\left(c_{5}+2\right)^{\lambda} \mathscr{R}_{s}^{\lambda} .
$$

But this means

$$
c_{1} /\left(2+c_{5}\right)^{\lambda}<2 c_{5}
$$

which is a contradiction if $c_{5}$ is sufficiently small. Hence, there exists at most one $v_{s} \in V$ such that

$$
\left|v_{s}-R_{s}\right|<c \mid \mathscr{R}_{s}^{\lambda} \text { if } c>0
$$

is sufficiently small. Thus, if the order of $f(z)$ is such that we have

$$
s^{s / k-s /(\rho+\varepsilon)} \leqq c / \mathscr{R}_{s}^{\lambda}
$$

then there will be at most one $v_{s} \in V$ to choose for $f^{\left(s_{l}\right)}\left(z_{l}\right)$. We therefore solve for the $\rho$ which satisfies (4.17). Using the definition of $\mathscr{R}_{s}$ given by (4.10) we have from (4.17)

$$
s^{s / k-s /(\rho+\varepsilon)} \leqq s^{-(s \lambda / k)(1-1 /(\rho+\varepsilon))}
$$

or

$$
1 / k-1 /(\rho+\varepsilon) \leqq-\lambda / k+\lambda / k(\rho+\varepsilon)
$$


which gives

$$
\rho+\varepsilon \leqq(k+\lambda) /(1+\lambda)
$$

or, since $\varepsilon$ was arbitrary,

$$
\rho \leqq(k+\lambda) /(1+\lambda)
$$

Hence, if $\rho<\rho_{0}=(k+\lambda) /(1+\lambda)$ then there exists an $\varepsilon>0$ and an $S$ so that (4.17) holds for all $s \geqq S$ and hence the coefficients $a_{s}$ are determined uniquely by the choice of $v_{1}, \ldots, v_{S-1}$. Since $V$ is countable there are at most countably many choices for the $a_{s}$ with $s<S$. Thus we get at most countably many entire functions of order $<\rho_{0}$ where $\rho_{0}=(k+\lambda) /(1+\lambda)$.

The proof remains valid if the condition on $V$ is weakened slightly, so that $\lambda$ can be defined as

$$
\lambda=-\liminf _{v, v^{\prime} \rightarrow \infty} \frac{\log \left|v-v^{\prime}\right|}{\log |v|}
$$

where $v, v^{\prime}$ are distinct elements of $V$.

THEOREM 4'. Let $\mathscr{F}$ be a family of entire functions as above where now $V$ satisfies

$$
\left|v-v^{\prime}\right|>c_{1} /(1+|v|)^{\lambda+\varepsilon(|v|)}
$$

for all $v \neq v^{\prime}$ and $\varepsilon(|v|) \rightarrow 0$ as $|v| \rightarrow \infty$. Then $\rho_{0}=(k+\lambda) /(1+\lambda)$ is a countable critical point of $\mathscr{F}$.

THEOREM 5. Let $\mathscr{F}$ be the family of entire functions with finite domain set, D, with $k$ elements and countable value set, $V$, such that

$$
\left|v-v^{\prime}\right|>c_{1} /(1+|v|)^{\lambda}
$$

for all $v \neq v^{\prime}, \lambda \geqq 0$. If $\rho=(k+\lambda) /(1+\lambda)$ then a countable critical point $\sigma_{0}$ for $\mathscr{F}$ is given by

$$
\sigma_{0}=\frac{k^{k /(k+\lambda)}(1+\lambda)}{(k+\lambda)\left|V_{k}\right|^{2 / k(1+\lambda)}}
$$

where $V_{k}$ is the Vandermonde determinant given by

$$
V_{k}=\prod_{i<j}^{k}\left(z_{j}-z_{i}\right)=\left|\begin{array}{cccc}
1 & z_{1} & \cdots & z_{1}^{k-1} \\
1 & z_{2} & \cdots & z_{2}^{k-1} \\
\vdots & & & \\
1 & z_{k} & \cdots & z_{k}^{k-1}
\end{array}\right| .
$$

Proof. Let $f(z)$ have the generalized Taylor series

$$
f(z)=\sum_{s=0}^{\infty} a_{s} P_{s}(z)
$$


where $P_{s}(z)=\prod_{i=1}^{k}\left(z-z_{i}\right)^{s_{i}}, s_{1}+\cdots+s_{k}=s$, and the $s_{l}$ are chosen so that

$$
s_{l}=\frac{s}{k}+c_{l} \frac{s / k}{\log (s / k)}+o\left(\frac{s}{\log s}\right) \text { with } \sum c_{l}=0
$$

We again have

and

$$
\begin{gathered}
f^{\left(s_{l}\right)}\left(z_{l}\right)=a_{s} s_{l} ! \prod_{i=1 ; i \neq l}^{k}\left(z_{l}-z_{i}\right)^{s_{i}}+R_{s} \\
R_{s}=\left[\sum_{\nu=s_{l}}^{s-1} a_{v} \frac{d^{s_{l}}}{d z^{s_{l}}}\left(P_{\nu}(z)\right)\right]_{z=z_{l}}
\end{gathered}
$$

and we once more seek an upper bound for $R_{s}$. (5.4) implies

$$
\left|R_{s}\right| \leqq\left[\sum_{v=s_{l}}^{s-1}\left|a_{v}\right|\left|\frac{d^{s_{l}}}{d z^{s_{l}}}\left(P_{v}(z)\right)\right|\right]_{z=z_{l}}
$$

Since $f(z)$ has order $\rho$ and type $\sigma$ we know for each $\varepsilon>0$ there exists $c>0$ such that

$$
\left|a_{v}\right| \leqq \frac{c \gamma_{1}^{v / \rho}}{\nu / \rho^{v / \rho}} \text { for all } \nu
$$

where $\gamma_{1}=\sigma e+\varepsilon$. Thus we get

$$
\left|R_{s}\right| \leqq \sum_{\nu=s_{l}}^{s-1} \frac{c \gamma_{1}^{\nu / \rho}}{\nu / \rho^{v / \rho}}\left|\frac{d^{s_{l}}}{d z^{s_{l}}}\left(P_{\nu}(z)\right)\right|_{z=z_{l}}
$$

Since

$$
\left|d^{s_{l}} / d z^{s_{l}}\left(P_{\nu}(z)\right)\right|_{z=z_{l}}
$$

is of the order of magnitude $c^{v}$ we see that the denominator of (5.7) dominates and as before we get the maximum of the summand at $\nu=s_{l}$ if $s$ is sufficiently large. Thus, we get

$$
\left|R_{s}\right| \leqq \frac{c_{1} s \gamma_{1}^{\left(s_{l} / \rho\right)} s_{l} !}{\left(s_{l} / \rho\right)^{s_{l} / \rho}}
$$

if $s \geqq S$.

Using (5.2) and Stirling's formula one can verify that

$$
\begin{aligned}
\gamma_{1}^{\left(s_{l} / \rho\right)} & =\gamma_{1}^{(s / k \rho)} e^{o(s)} \\
s_{l} ! & =\left(\frac{s}{k}\right)^{s / k} \exp \left[c_{l}(s / k)\right] e^{-(s / k)} e^{o(s)} \\
\left(\frac{s_{l}}{\rho}\right)^{s_{l} l \rho} & =\left(\frac{s}{k}\right)^{s / k \rho} \exp \left[c_{l}(s / k \rho)\right]\left(\frac{1}{\rho}\right)^{s / k \rho} e^{o(s)} .
\end{aligned}
$$

These last three relations together with (5.8) imply

$$
\left|R_{s}\right| \leqq \gamma_{1}^{s / k \rho}\left(\frac{s}{k}\right)^{s / k}\left(\frac{s}{k \rho}\right)^{-s / o k} \exp \left(c_{l}\left[\frac{s}{k}\left(1-\frac{1}{\rho}\right)\right]\right) e^{-s / k} e^{o(s)}=\mathscr{R}_{s}
$$


Essentially repeating the argument used in Theorem 4 where now

$$
\begin{aligned}
\left|v_{s}-R_{s}\right| & =\left|a_{s} s_{l} ! \prod_{i=1 ; i \neq l}^{k}\left(z_{l}-z_{i}\right)^{s_{i}}\right| \\
& <\gamma_{1}^{s / \rho}\left(\frac{s}{\rho}\right)^{-s / \rho}\left(\frac{s}{k}\right)^{s / k} \exp \left(\frac{s}{k}\left(c_{l}-1\right)\right)\left(\prod_{i=1 ; i \neq l}^{k}\left|z_{l}-z_{i}\right|\right)^{s / k} e^{o(s)}=b_{s}
\end{aligned}
$$

we get

$$
b_{s} \leqq c / \mathscr{R}_{s}^{\lambda}
$$

and we solve for the $\sigma$ which satisfies (5.14).

Now, (5.12), (5.13) and (5.14) together imply

$$
\begin{gathered}
\gamma_{1}^{-\lambda s / k \rho}\left(\frac{s}{k}\right)^{-\lambda s / k}\left(\frac{s}{\rho}\right)^{\lambda s / \rho k} \exp \left(-\lambda c_{l}\left[\frac{s}{k}\left(1-\frac{1}{\rho}\right)\right]\right) e^{\lambda s / k}\left(\frac{1}{k}\right)^{\lambda s / k \rho} \\
\geqq \gamma_{1}^{s / \rho}\left(\frac{s}{\rho}\right)^{-s / \rho}\left(\frac{s}{k}\right)^{s / k} e^{-s / k} e^{c_{l} s / k}\left(\prod_{i=1 ; i \neq l}^{k}\left|z_{l}-z_{i}\right|\right)^{s / k}
\end{gathered}
$$

By noting that $\rho=(k+\lambda) /(1+\lambda), \sum c_{l}=0$ and taking the product of both sides of inequality (5.15) we have

$$
\frac{1+\lambda}{k+\lambda} e k^{k /(k+\lambda)} \geqq \gamma_{1} \gamma^{1 / k(1+\lambda)}
$$

where $\gamma=\left|V_{k}\right|^{2}$ since

$$
\left|\prod_{l=1}^{k}\left(\prod_{i=1 ; i \neq l}^{k}\left(z_{l}-z_{i}\right)\right)\right|=\left|V_{k}\right|^{2} .
$$

From $\gamma_{1}=\sigma e+\varepsilon$ and (5.16) we get

$$
\sigma \leqq \frac{k^{k /(k+\lambda)}(1+\lambda)}{(k+\lambda)\left|V_{k}\right|^{2 / k}(1+\lambda)}=\sigma_{0}
$$

Hence, if $\rho=(k+\lambda) /(1+\lambda)$ and $\sigma<\sigma_{0}$ there is a unique choice for $f^{\left(s_{l}\right)}\left(z_{l}\right)$ if $s \geqq S$ and we again get at most a countable number of functions of order $(k+\lambda) /(1+\lambda)$ and type $\sigma$.

The condition on the value set, $V$, could be modified to read for all $v \neq v^{\prime} \in V$,

$$
\left|v-v^{\prime}\right|>\frac{c_{1}}{(1+|v|)^{\lambda+(\lambda / \log \log |v|)+\varepsilon(|v|)}}
$$

where $\varepsilon(|v|)=o(1 / \log \log |v|)$. The same methods of Theorem 5 could then be used to derive another countable critical point $\sigma_{0}^{\prime}=\sigma_{0}^{\prime}\left(k, \lambda, \lambda_{1}\right)$.

THEOREM 6. Let $\mathscr{F}$ be the family of entire functions with finite domain set, $D$ $=\left\{z_{1}, \ldots, z_{k}\right\}$, and a value set, $V$, such that for each $z \in C$ there exists $v \in V$ with

$$
|v-z|<c_{2} /(1+|z|)^{\mu}
$$


where $-1<\mu<0$ and $c_{2}>0$. Then $\rho_{1}=(k+\mu) /(1+\mu)$ is an uncountable critical point of $\mathscr{F}$. If for all $f \in \mathscr{F}$, ord $f=(k+\mu) /(1+\mu)$ then

$$
\sigma_{1}=\frac{k^{k /(k+\mu)}(1+\mu)}{(k+\mu)\left|V_{k}\right|^{2 / k(1+\mu)}}
$$

is an uncountable critical point of $\mathscr{F}$.

Proof. Let

$$
f(z)=\sum_{s=0}^{\infty} a_{s} P_{s}(z)
$$

where $P_{s}(z)=\prod_{i=1}^{k}\left(z-z_{i}\right)^{s_{i}}$ and $s_{1}+s_{2}+\cdots+s_{k}=s$. Choosing the $s_{l}$ as before we know from Theorem 4 that

$$
f^{\left(s_{l}\right)}\left(z_{l}\right)=a_{s} s_{l} ! \prod_{i \neq l}^{k}\left(z_{l}-z_{i}\right)^{s_{i}}+R_{s}
$$

and

$$
R_{s}=\sum_{\nu=s_{l}}^{s-1} a_{v} s_{l} ! \sum_{\sum \lambda_{l}=s_{l}: \sum v_{i}=v} \prod_{i \neq l}\left(\begin{array}{c}
\nu_{i} \\
\lambda_{i}
\end{array}\right)\left(z_{l}-z_{i}\right)^{s_{i}} .
$$

Since $R_{s}$ depends on $a_{i}$ with $i<s$, if we can ensure at least two choices for $f^{\left(s_{l}\right)}\left(z_{l}\right)=v_{s}$ we see from (6.2) that we will have at least two choices for $a_{s}$. Thus, we would like to choose the $v_{s}$ in at least two ways so that the following inequalities are satisfied:

$$
\left|a_{s}\right|<\gamma_{1}^{s} s^{-s / \rho}
$$

and

$$
\left|R_{s}\right| \leqq s^{s(1-s / \rho) / k} \gamma_{2}^{s}=\mathscr{R}_{s}
$$

where

$$
\gamma_{2}=\gamma_{1} c_{4}
$$

and

$$
\gamma_{1}>\left(c c_{2}^{\prime} /\left(2 c_{4}\right)^{\mu} c_{3}\right)^{1 /(1+\mu)}
$$

with $c_{2}^{\prime}=\max \left\{c_{2}, 1\right\}$ and where $c>0$ is determined below. The positive constants $c_{3}$ and $c_{4}$ depend on the domain set $D$. That is, $c_{3}$ is chosen such that

$$
s_{l} ! \prod_{i \neq l}\left|z_{l}-z_{i}\right|^{s_{i}}>s^{s / k} c_{3}^{s}>0
$$

and $c_{4}$ is chosen so that

$$
\begin{aligned}
\left|R_{s}\right| & <\sum_{v=s_{l}}^{s-1}\left|a_{s_{l}}\right| s^{s / k}\left(k^{-(1 / k)}\right)^{s} c_{3}^{s} \leqq c_{4}^{s} \gamma_{1}^{s} s^{-s(1-1 / \rho) / k} \\
& =\gamma_{2}^{s} s^{s(1-1 / \rho) / k}
\end{aligned}
$$


The upper bound for $\left|R_{s}\right|$ given by (6.8) is determined using exactly the same reasoning as in Theorem 4.

By hypothesis there is at least one $v_{s} \in V$ with

$$
\left|v_{s}-R_{s}\right|<c_{2} /\left(1+\left|R_{s}\right|\right)^{\mu}=r .
$$

We now show that for $c(>0)$ sufficiently large, there exists at least two $v_{s} \in V$ such that

$$
\left|v_{s}-R_{s}\right|<c r_{1}
$$

where $r_{1}=c_{2}\left(1+\mathscr{R}_{s}\right)^{-\mu}$. For sufficiently large $R_{s}$, say $\left|R_{s}\right| \geqq R_{0}$, we will have $\left|R_{s}\right|>r$. We can then choose $R_{s}^{\prime}$ so that $\left|R_{s}^{\prime}\right|=\left|R_{s}\right|$ and $\left|R_{s}-R_{s}^{\prime}\right|=2 r$. There will then be at least two $v_{s} \in V$ satisfying

$$
\left|v_{s}-R_{s}\right|<3 r \leqq 3 r_{1}
$$

(cf. Figure 6-1).

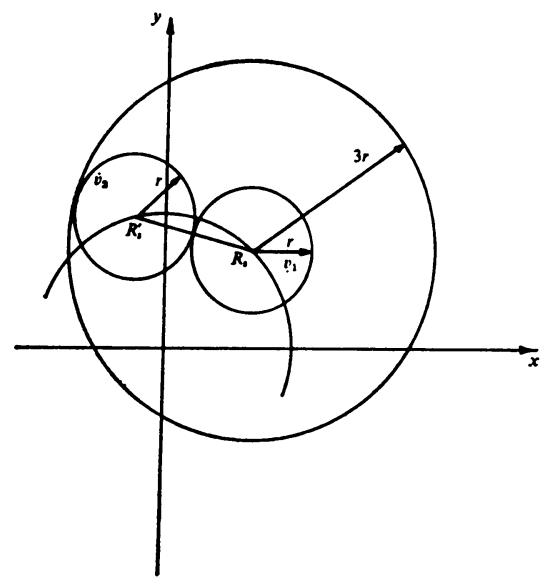

FIGURE 6-1

By our assumption on the value set, $V$, it follows that there are infinitely many $v \in V$. Thus, we choose $R_{0}^{\prime}>R_{0}$ and so that there are at least two $v$ 's inside the circle $|z|=R_{0}^{\prime}$. Then if $\left|R_{s}\right|<R_{0}$ there will be at least two $v$ 's in $V$ satisfying

$$
\left|v_{s}-R_{s}\right|<2 R_{0}^{\prime}
$$

(cf. Figure 6-2).

Hence, there will be at least two $v_{s} \in V$ such that

$$
\begin{aligned}
\left|v_{s}-R_{s}\right|<3 r_{1} & \text { if }\left|R_{s}\right| \geqq R_{0}, \\
<2 R_{0}^{\prime} & \text { if }\left|R_{s}\right|<R_{0} .
\end{aligned}
$$

We now choose

$$
c>\max \left\{2 R_{0}^{\prime} / c_{2}, 3\right\} \text {. }
$$




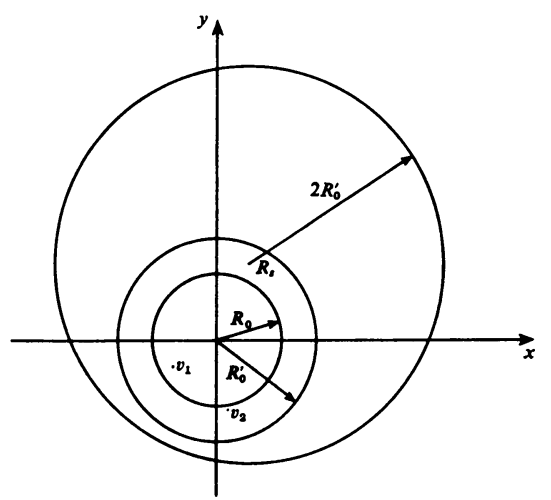

FIGURE 6-2

Then since $\left(1+\mathscr{R}_{s}\right)^{-\mu} \geqq 1$ we will have at least two $v_{s} \in V$ for which (6.10) holds as asserted.

From (6.2) and (6.10) we see that we will have at least two choices of $f^{\left(s_{l}\right)}\left(z_{l}\right)=v_{s}$ such that

$$
\left|a_{s}\right|=\left|v_{s}-R_{s}\right| /\left(s_{l} ! \prod_{i \neq l}\left|z_{l}-z_{i}\right|^{s_{i}}\right)<c r_{1} / s^{s / k} c_{3}^{s} .
$$

Since $-1<\mu<0,(6.5)$ implies

$$
\mathscr{R}_{s}^{-\mu} \geqq\left|R_{s}\right|^{-\mu} \text {. }
$$

Hence, we can rewrite (6.14) as

$$
\begin{aligned}
\left|a_{s}\right| & <s^{-s / k}\left(c_{3}^{-1}\right)^{s} c c_{2}\left(2 \mathscr{R}_{s}\right)^{-\mu} \\
& =s^{-s / k}\left(c_{3}^{-1} c c_{2}^{\prime} 2^{-\mu}\right)^{s} \mathscr{R}_{s}^{-\mu} \\
& =\left(c c_{3}^{-1} c_{2}^{\prime} 2^{-\mu}\right)^{s} s^{-s(1+\mu(1-1 / \rho)) / k} \gamma_{2}^{-\mu s}
\end{aligned}
$$

where $c_{2}^{\prime}=\max \left\{c_{2}, 1\right\}$. Inequality (6.4) will therefore be satisfied for at least two possible choices of $a_{s}$ if

$$
\left(c c_{3}^{-1} c_{2}^{\prime} 2^{-\mu}\right)^{s} s^{-s(1+\mu(1-1 / \rho)) / k} \gamma_{2}^{-\mu s}<\gamma_{1}^{s} s^{-s / \rho} .
$$

If we let $\rho=(k+\mu) /(1+\mu)$ in (6.17) we see that (6.4) will hold for all $s$ by our choice of $\gamma_{1}$ and $\gamma_{2}$. Hence, there are $2^{\aleph_{0}}$ possibilities for functions of the form (6.1) and $\rho_{1}=(k+\mu) /(1+\mu)$ is an uncountable critical point for $\mathscr{F}$.

We now assume $\rho=\rho_{1}$. Choosing

$$
s_{l}=\frac{s}{k}+c_{l} \frac{s / k}{\log (s / k)}+o\left(\frac{s}{\log s}\right)
$$


as in Theorem 5, we can repeat the above argument to get an uncountable critical point for the type equal to

$$
\sigma_{1}=\frac{k^{k /(k+\mu)}(1+\mu)}{(k+\mu)\left|V_{k}\right|^{2 / k(1+\mu)}}
$$

where we now have two choices for $a_{s}$ which satisfy

$$
\left|a_{s}\right|<\gamma_{1}^{s / \log s}\left(\frac{s}{\rho}\right)^{-s / \rho}(\sigma e)^{s / \rho}
$$

and

$$
\left|R_{s}\right| \leqq(\sigma e)^{s / k \rho}\left(\frac{s}{k}\right)^{s / k}\left(\frac{s}{k \rho}\right)^{-s / k \rho} \exp \left(c_{l}\left[\frac{s}{k}\left(1-\frac{1}{\rho}\right)\right]\right) e^{-s / k} \gamma_{2}^{s / \log s}
$$

for the proper choices of the positive constants $\gamma_{1}$ and $\gamma_{2}$.

ACKNOWLedgment. The author would like to thank Professor E. G. Straus who directed this research and contributed many helpful suggestions.

\section{REFERENCES}

1. R. P. Boas, Jr., Entire functions, Academic Press, New York, 1954.

2. R. C. Buck, Integral valued entire functions, Duke Math. J. 15 (1948), 879-891.

3. G. H. Hardy, On a theorem of Mr. G. Pólya, Proc. Cambridge Philos. Soc. 19 (1916-1919), 60-63.

4. G. Pólya, Über Ganzwertige Ganze Funktionen, Rend. Circ. Mat. Palermo 40 (1915), 1-16.

5. E. G. Straus, On entire functions with algebraic derivatives at certain algebraic points, Ann. of Math. 52 (1950), 188-198.

6. D. Sato, Integer valued entire functions, Dissertation, University of California, Los Angeles, 1961.

7. D. Sato and E. G. Straus, Generalized interpolation by analytic functions, J. Math. Sci. 1 (1966), 53-76.

Control Data Corporation,

Los ANgeles, California 